\title{
Progressive retinal dystrophy due to retinol transport defect
}

INSERM

\section{Source}

INSERM. (1999). Orphanet: an online rare disease and orphan drug data base. Progressive retinal dystrophy due to retinol transport defect. ORPHA:352718

Progressive retinal dystrophy due to retinol transport defect is a rare, genetic, metabolite absorption and transport disorder characterized by progressive rod-cone dystrophy, usually presenting with impaired night vision in childhood, progressive loss of visual acuity and severe retinol deficiency without keratomalacia. Association with ocular colobomas, severe acne and hypercholesterolemia has been reported. 